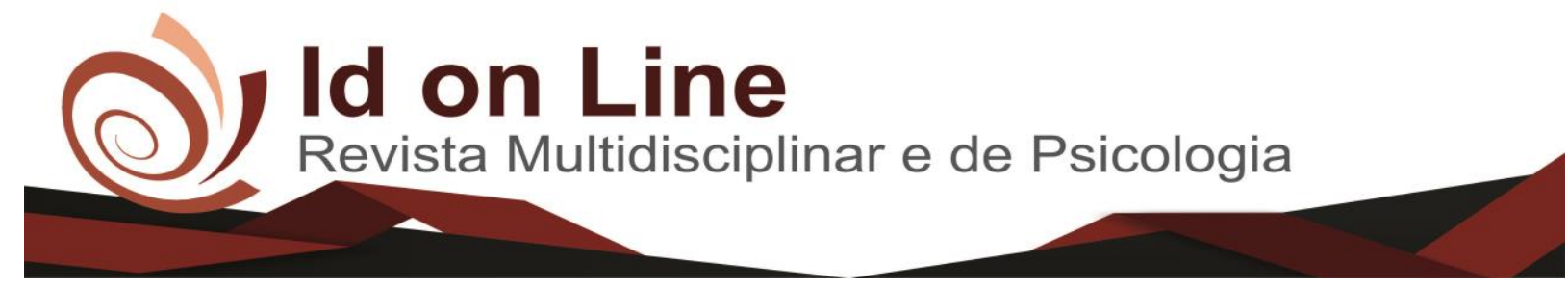

Artigo

\title{
Avaliação do Perfil da Assistência Farmaceutica dos Munícipios do Sudoeste da Bahia
}

\author{
Silvana Rocha Teixeira ${ }^{\text {; }}$ Mauro Fernandes Teles² ; Pablo Maciel Brasil Moreira ${ }^{3}$
}

\begin{abstract}
Resumo: Com a Política Nacional de Medicamentos a Assistência Farmacêutica passa a ser compreendida como ponto complementar nas práticas desenvolvidas para promoção, prevenção e recuperação à saúde, ultrapassando a simples aquisição e distribuição de medicamentos. Entretanto os municípios brasileiros encontram dificuldades para cumprir as diretrizes dessa política. Este estudo objetiva avaliar a capacidade de gestão da Assistência Farmacêutica dos municípios vinculados a Microrregião de Saúde do Sudoeste Baiano. Trata-se de um estudo de corte transversal quali-quantitativo, realizado com os 12 municípios vinculados a Microrregião Região de Saúde do Sudoeste da Bahia que aceitaram participar da pesquisa por meio da aplicação de questionário virtual, adaptado. Os resultados mostram que os farmacêuticos que trabalham na Assistência Farmacêutica dos municípios possuem uma faixa salarial que varia entre 1300 a 4000 reais, com carga horária de 20 a 40 horas semanal, onde $50 \%$ deles possuem um segundo vínculo empregatício. A maioria dos municípios encontra dificuldade em realiza gestão técnica, não possui uma Comissão de Farmácia e Terapêutica (CFT), não utilizam Manual de Boas práticas de Armazenamento e Distribuição de medicamentos nem POP's (Protocolos Operacional Padrão). Desmontaram objeção para cumprir as etapas do ciclo da Assistência Farmacêutica e garantir o uso racional de medicamentos da população. Conclui-se então que apesar dos avanços ocorridos na consolidação do SUS, em particular na Assistência Farmacêutica, os municípios ainda se deparam com dificuldades, em gerenciar e executar ações básicas que envolvem a Assistência Farmacêutica o que pode levar a deficiência no acesso da população ao medicamento.
\end{abstract}

Palavras Chave: Assistência Farmacêutica; Avaliação; Medicamentos.

\section{Profile Assessment of Pharmaceutical Care of Bahia Southwest Municipalities}

\begin{abstract}
With the National Drug Policy, Pharmaceutical Assistance is understood as a complementary point in the practices developed for health promotion, prevention and recovery, going beyond the simple acquisition and distribution of medicines. However, Brazilian municipalities find it difficult to comply with the guidelines of this policy. This study aims to evaluate the management capacity of the Pharmaceutical Assistance of the municipalities linked to the Health Micro-region of Southwest Bahia. This is a qualitative and quantitative crosssectional study carried out with the 12 municipalities linked to the Southwest Health Region of Bahia, which accepted to participate in the study through the application of an adapted virtual questionnaire. The results show that the pharmacists who work in the Pharmaceutical Assistance of the municipalities have a salary range ranging from 1300 to 4000 reais, with a workload of 20 to 40 hours per week, where $50 \%$ of them have a second employment relationship. Most municipalities find it difficult to carry out technical management, do not have a Pharmacy and Therapeutics Commission (CFT), do not use a Manual of Good Practices in Storage and Distribution of Medicines and POPs (Standard Operational Protocols). They objected to complying with the stages of the Pharmacy Assistance cycle and ensuring the rational use of medicines by the population. It is concluded that despite the progress made in the consolidation of the SUS, particularly in Pharmaceutical Assistance, municipalities still face difficulties in managing and executing basic actions involving Pharmaceutical Assistance, which may lead to deficiency in the population's access to product.
\end{abstract}

Keywords: Pharmaceutical Assistance; Evaluation; Medicines.

\footnotetext{
${ }^{1}$ Graduanda em Farmácia, Faculdade Independente do Nordeste-FAINOR, Vitória da Conquista, Bahia, Brasil.

Contato: Silvana Rocha Teixeira, e-mail: silvanasrt41@gmail.com. Telefone: (77) 9.91204341;

${ }^{2}$ Farmacêutico, Mestre em Saúde Pública, Docente da Faculdade Independente do Nordeste-FAINOR, http://www.fainor.com.br, Vitória da Conquista, Bahia, Brasil.

${ }^{3}$ Farmacêutico pela Universidade Federal da Bahia, Especialista em Gestão da Assistência Farmacêutica pela Universidade Federal de Santa Catarina - UFSC
} 


\section{Introdução}

O SUS foi regulamentado pela Lei Orgânica da Saúde 8080/90, que criou dispositivos que as orientam as ações básicas, direcionamento quanto à gestão, competência, atribuições e financiamento de cada esfera do governo, reorientação do processo de formação de recursos humanos, assegurar à profusão da assistência terapêutica integral, englobando a Assistência Farmacêutica (AF), além de enfatiza a necessidade de formulação da política de medicamentos. (BRASIL, 1990)

Até o final da década de 90 a AF foi ocasionalmente desenvolvida pela Central de Medicamentos (CEME), instituída em 1975 extinta com a elaboração e publicação da Política Nacional de Medicamentos (PNM) em 1998, seguindo os princípios e diretrizes do SUS. (BRASIL, 1971). A PNM trouxe o primeiro conceito oficial da AF, definindo-a como parte integrante de um conjunto de práticas direcionada para a promoção, prevenção e recuperação de saúde, contemplando atividades que ultrapassa o simples abastecimento de medicamentos. (BRASIL, 1998; CAMPENSE, 2006)

No âmbito do financiamento da AF, somente a partir do final da década de 1990, ficou determinada na Portaria GM no . 176/99 que financiamento seria norteado pelo incentivo à Assistência Farmacêutica Básica e a participação do nível federal seria vinculada ao repasse fundo a fundo. Com isso os municípios passaram a coordenar a aquisição de medicamentos essenciais distribuídos na Assistência Farmacêutica Básica. (BRASIL, 1999)

Com a PNM ficou estabelecido às diretrizes, as prioridades e as responsabilidades da AF para os gestores das três esferas de governo. Uma das diretrizes dessa política é a reorientação da $\mathrm{AF}$ que tem por finalidade o desenvolvimento de atividades relacionadas à promoção do acesso da população aos medicamentos essenciais e não deve se limitar apenas à aquisição e distribuição de medicamentos (BRASIL, 1998).

Fruto dos avanços nas discussões sobre o tema, em 2004 foi elaborada da PNAF Política Nacional de Assistência Farmacêutica como parte integrante da Política Nacional de Saúde, sendo aprovada pelo Conselho Nacional de Saúde e publicada pela Resolução CNS 338/2004 e seus princípios englobaram um conjunto de ações voltadas à promoção, proteção e recuperação da saúde e garantindo os princípios da universalidade, integralidade e equidade. Destaca-se como política pública norteadora para formulação de políticas setoriais, dentre elas, a formação de recursos humanos formados para essa nova concepção. (BRASIL, 2004) 
Entretanto, segundo Fraga et al (2005), ainda é incipiente a Assistência Farmacêutica desenvolvida nos municípios brasileiros, uma vez que preservam a ênfase na aquisição de medicamentos. Além disso, os municípios ainda não são capazes de garantir os propósitos maiores desta política, como a necessária segurança, eficácia e qualidade, a promoção do uso racional e o acesso da população aos medicamentos essenciais. (NASCIMENTO JR, 2000)

Deste modo, a Assistência Farmacêutica nos municípios brasileiros ainda não possui condições suficientes para assumirem as funções de executores desta política. Problemas na organização das atividades relacionadas ao medicamento, são apontados em estudos sobre a implementação da PNM, estes problemas são decorrentes da falta de prioridade em relação a este campo da Assistência. (BARRETO e GUIMARAES, 2010)

Para Mayorga et al (2004), que analisou a situação da assistência farmacêutica no Brasil, os municípios e estados enfrentam problemas relacionados ao desenvolvimento e qualidade dos serviços farmacêuticos, debilidade na infraestrutura e operacionalidade, além da dificuldade no atendimento da demanda populacional por medicamentos.

Nesta perspectiva, este estudo tem por objetivo avaliar a capacidade de gestão da assistência farmacêutica nos municípios vinculados á microrregião de Saúde do Sudoeste Baiano, com destaque para a organização das ações e serviços destes municípios.

\section{Metodologia}

Trata-se de um estudo de corte transversal quali-quantitativo, que se apresenta dentro de uma abordagem de cunho descritivo exploratório. O estudo foi realizado com os 19 municípios vinculados a Microrregião de Saúde do Sudoeste da Bahia, conforme Lei Estadual 13.204/2014 do Estado da Bahia. Os profissionais farmacêuticos dos municípios que fizeram parte da amostra desse estudo foram convidados a participar por meio do endereço oficial de email da Assistência Farmacêuticos Municipais cadastrados na Secretaria Estadual de Saúde do Estado da Bahia. A coleta de dados foi realizada por meio da aplicação de questionário virtual, conforme instrumento indicado no Anexo I, adaptado de Barreto e Guimarães (2010) e Freitas et. al. (2016). Foi realizado um piloto para aplicação do instrumento e correção das inconsistências. 
Foram avaliadas as variáveis; Capacidade para realizar a gestão técnica da Assistência Farmacêutica; Capacidade para trabalhar com uma Relação de Medicamentos Essenciais ou para elaborá-la; Capacidade para assegurar a programação e a aquisição de medicamentos em quantidades adequadas e tempo oportuno; Capacidade para assegurar o acesso a medicamentos de qualidade e seguros quanto à manutenção das suas características físico-químicas e terapêuticas; Capacidade para realizar a dispensação adequada de medicamentos nas farmácias e/ou unidades dispensadoras nas unidades de saúde; Capacidade para dispor de Recursos Humanos qualificados e em número suficiente para a Assistência Farmacêutica e Capacidade para implantar ações de farmacovigilância no município. Os dados obtidos foram tabulados e analisados por meio de estatística descritiva e do software IBM® SPSS Statistics 22.

Conforme Resolução do Conselho Nacional de Saúde n 466/2012 e Resolução do Conselho Nacional de Saúde $n^{\circ}$ 510/2016, a coleta de dados se iniciou após a aprovação do projeto pelo Comitê de Ética em pesquisa do Hospital Municipal Esaú Matos, CAAE no 72079617000008089. Após o aceite em participar da pesquisa, foram convidados a assinar o Termo de Consentimento Livre e Esclarecido (TCLE) constante no Anexo II, em seguida tendo acesso ao instrumento de coleta de dados para prosseguir com seu preenchimento.

\section{Resultados e Discursões}

A Assistência Farmacêutica é entendida como um conjunto de atividades voltadas para a promoção, proteção e recuperação á saúde, objetivando o uso racional de medicamentos. Este conjunto envolve a seleção, programação, aquisição, distribuição e dispensação de medicamentos, garantindo a qualidade dos produtos e serviços, assim como avaliação de sua utilização, no alcance da melhoria da qualidade de vida da população. (BRASIL, 2004). O farmacêutico é visto como um dos responsáveis pela qualidade de vida da população, uma vez que é o profissional que abrange as melhores condições para a garantia da qualidade dos medicamentos, de forma que sem esse profissional o programa de assistência farmacêutica não obteria sucesso (BASILE, 2008).

A pesquisa foi realizada com 12 farmacêuticos, ligados á assistência farmacêutica de 12 municípios vinculados a microrregião de Vitória da Conquista. 
Tabela 1. Análise do vinculo empregatício, carga horária e remuneração dos Farmacêuticos que atuam na Assistência Farmacêutica dos municípios vinculados a microrregião de saúde de Vitória da Conquista.

\section{Variáveis}

\section{Local de trabalho}

\begin{tabular}{|c|c|c|}
\hline Assistência Farmacêutica Municipal & 12 & 100,0 \\
\hline \multicolumn{3}{|l|}{ Qual vínculo } \\
\hline Municipal & 6 & 50,0 \\
\hline Privado & 6 & 50,0 \\
\hline \multicolumn{3}{|l|}{ Tipo de vínculo } \\
\hline Concurso & 1 & 8,3 \\
\hline Contrato & 11 & 91,7 \\
\hline \multicolumn{3}{|l|}{ Carga horária } \\
\hline $20 \mathrm{hrs}$ & 7 & 58,3 \\
\hline $30 \mathrm{hrs}$ & 1 & 8,3 \\
\hline $40 \mathrm{hrs}$ & 4 & 33,3 \\
\hline \multicolumn{3}{|l|}{ Remuneração bruta } \\
\hline $\mathrm{R} \$ 1.300,00$ & 1 & 8,3 \\
\hline $\mathrm{R} \$ 1.500,00$ & 3 & 25,0 \\
\hline $\mathrm{R} \$ 1.800,00$ & 1 & 8,3 \\
\hline $\mathrm{R} \$ 2.000,00$ & 1 & 8,3 \\
\hline $\mathrm{R} \$ 2.300,00$ & 2 & 16,7 \\
\hline $\mathrm{R} \$ 2.980,00$ & 1 & 8,3 \\
\hline $\mathrm{R} \$ 3.000,00$ & 1 & 8,3 \\
\hline $\mathrm{R} \$ 4.000,00$ & 2 & 16,7 \\
\hline Total & 18 & 100,0 \\
\hline
\end{tabular}

Fonte: Pesquisa própria

Na tabela 1 podemos observar que o salário dos farmacêuticos que trabalha na gestão pública, dos municípios que participaram da pesquisa, pode variar entre 1300 á 4000 reais, com uma carga horária de 20 a 40 horas semanais, o que pode está relacionado á necessidade de $50 \%$ deles possuírem 2 vínculos empregatício. Deve-se levar em consideração, também, o fato de que $91,7 \%$ dos farmacêuticos possuem um tipo de vinculo precário, através de contrato, com a Assistência Farmacêutica do município, apenas 8,3\% dos municípios, o farmacêutico é contratado por meio de concurso público. Vale salientar que o segundo vinculo dos farmacêuticos é em drogaria, onde a grande maioria é contratada com base na CLT (Consolidação das Leis de Trabalho), conforme Lei № 5.452/43. 
O porte da maioria destes municípios, cerca de 58,3\%, como evidenciado na tabela 2, é de até 15000 habitantes como, nos quais contam com uma média de 1,29 farmacêuticos ligados á Assistência Farmacêutica em cada município, visto de forma positiva de acordo com a WHO, que recomenda para cada 20000 habitantes, no mínimo 1 farmacêutico.

Tabela 2. Análise do porte dos municípios vinculados a microrregião de saúde de Vitória da Conquista e média dos farmacêuticos ligados á Assistência Farmacêutica.

\begin{tabular}{lcc}
\multicolumn{1}{c}{ Porte do Município } & $\mathbf{n}(\%)$ & $\begin{array}{c}\text { Farmacêuticos ligados a } \\
\text { A.F. (X) }\end{array}$ \\
Até 15.000 habitantes & $7(58,3)$ & 1,29 \\
De 15.001 a 25.000 habitantes & $1(8,3)$ & 1,00 \\
De 25.001 a 50.000 habitantes & $3(25,0)$ & 2,33 \\
Maior que 100.000 habitantes & $1(8,3)$ & 13,00 \\
Valor p* & & 0,019 \\
\hline
\end{tabular}

Fonte: Pesquisa própria

A pesquisa em questão aponta para uma deficiência na capacidade de realizar a gestão técnica dos municípios, uma vez que, as ações de organização da AF na maioria dos municípios não estão integradas ou estão integradas parcialmente nas ações de saúde. Em uma pesquisa feita pela Organização Pan-Americana da Saúde (Opas), em parceria com o Ministério da Saúde (Opas, 2005), demonstrou lacunas na gestão e na integração das ações da Assistência Farmacêutica às ações de saúde, evidenciando a indispensabilidade de investir para qualificação dos serviços.

Acompanhar e avaliar de maneira constante as atividades desenvolvidas faz parte de uma estratégia para fortalecer o modelo da Assistência Farmacêutica. Entretanto a maioria dos municípios em questão não costuma avaliar os resultados da assistência farmacêutica.

A Comissão de Farmácia e Terapêutica (CFT) tem como objetivo selecionar os medicamentos essenciais utilizados no sistema de saúde, além de auxiliar na gestão de questões ligadas á Assistência Farmacêutica. É composta, por profissionais de saúde de diversas formações, entre eles o farmacêutico, que tem como papel principal realizar medidas educativas para promover o uso racional de medicamentos. A CFT consiste em uma estratégia onde o gestor toma decisões mais consistentes segundo diretrizes estabelecidas. (BARRETO; GUIMARÃES, 2010) 
Em relação á seleção de medicamentos realizada pelos municípios, a pesquisa aponta para inexistência de uma Comissão de Farmácia e Terapêutica (CFT) em 83\% dos municípios entrevistados, na mesma proporção que não possuem uma relação de medicamentos própria do município e sim uma lista de medicamentos recebidos por repasse estadual e federal, o que pode acarretar ao não atendimento a demanda local de saúde. Problemas como este tem levado á problemas no acesso da população aos medicamentos acarretando em um aumento de ações judiciais contra o SUS.

Em 2005 foi realizado, na cidade de São Paulo, um estudo que analisou as ações judiciais levantadas por usuários da cidade que solicitaram o acesso a medicamentos gratuitos pelo SUS, onde foi constatado que $62 \%$ dos medicamentos solicitados faziam parte da Relação Municipal de Medicamentos Essenciais, entretanto, $73 \%$ dos medicamentos solicitados, que não eram padronizados, poderiam ter sido substituídos por medicamentos padronizados no município, com isso a maioria das ações poderiam ter sido evitada se fossem consideradas as diretrizes do SUS e a observância das relações de medicamentos essenciais (VIEIRA, 2007).

Em paralelo á isso a pesquisa constata que em grande parte dos municípios não existe divulgação, da lista de medicamentos selecionados para os prescritores, nem a realização de ações para a promoção, dos mesmos, para adesão á REMUME, o que pode levar a dificuldade no acesso ao medicamento e consequentemente deficiência na promoção do uso racional de medicamentos.

A Programação de medicamentos é uma etapa de grande importância do ciclo da Assistência Farmacêutica, que consiste em atender á uma demanda de serviços através de uma estimativa de quantidades a serem adquirida, definindo um período de tempo, levando a uma influência direta sobre o abastecimento e o acesso ao medicamento. (BRASIL, 2002)

A pesquisa aponta para problemas na programação dos municípios, a grande maioria deles encontra dificuldades para realização de uma programação apropriada devido à falta de um meio sistematizado, uma vez que o método manual não aponta dados confiáveis, em paralelo a isso, o farmacêutico, não participa do processo de compra dos medicamentos, apenas indica o que é necessário ser comprado e a quantidade. Não existe uma analise de consumo histórico, de consumo ajustado e oferta de serviços e recursos financeiros, o que leva a uma aquisição de medicamentos não satisfatória para atender as demandas dos municípios.

A falta do farmacêutico na programação gera um conjunto de impasses na gestão da Assistência Farmacêutica levando ao improviso e ao não cumprimento de recomendações técnicas. (Marin et al, 2003) 
Para BRUS, et al (2014) as deficiências na etapa de programação da gestão podem acarretar riscos de desperdício e de desabastecimento, levado ao comprometimento das demais etapas do Ciclo da Assistência Farmacêutica, levando a transtornos para a população, devido a falta de acesso aos medicamentos necessários.

A aquisição de medicamentos realizada na grande maioria dos municípios participantes da pesquisa aponta para irregularidades, uma vez que na maioria dos municípios não existe nenhuma política e normas de aquisição de medicamentos. Existem alguns municípios que adquirem os medicamentos de forma direta, sem licitação, entretanto a maioria realiza licitação, porém sem SRP (Sistema de Registro de Preço).

A aquisição de medicamentos é representada como uma das etapas do Ciclo da Assistência Farmacêutica, na qual constitui um conjunto de orientações planejadas que tem como objetivo a seleção do licitante que possui a melhor proposta para atender uma determinada necessidade. Ela tem como propósito, o abastecimento de medicamentos com quantidade e qualidade ideal, no menor custo disponível, dentro do encontrado no mercado promovendo uma terapia racional, em espaço e tempo determinados (MARIN, 2003).

De acordo com o art. 3o da Lei no 8.666, de 1993, o procedimento licitatório objetiva garantir a seleção da melhor proposta para a administração pública, na qual deve está em plena concordância com os princípios básicos da legalidade, da impessoalidade, da moralidade, da publicidade e da probidade administrativa. A “economicidade” representa um dos princípios consagrados, tendo em vista que o procedimento licitatório pretende selecionar a melhor proposta para a administração pública (BRASIL, 1993).

Em relação ao armazenamento dos medicamentos os municípios demonstram debilidade no processo, apontando para más condições sanitárias, inexistência de uma rotina adequada para conservação do local, muitos não fazem uso de condicionadores de ar, ventiladores ou exaustores, nem registro de controle diário de temperatura. Destaca-se também a falta de Manual de Boas praticas de Armazenamento e Distribuição de medicamentos, bem como POP's (Protocolos Operacional Padrão) e Plano de Gerenciamento de Resíduos de Serviços de Saúde - PGRSS em grande parte dos municípios participantes da pesquisa. As maiorias dos municípios não contam com transporte próprio para a distribuição adequada dos medicamentos.

De acordo com as Diretrizes para estruturação de farmácias no âmbito do Sistema Único de Saúde (2009), os produtos farmacêuticos devem ser armazenados cumprindo às condições técnicas ideais de luminosidade, temperatura e umidade, objetivando assegurar a manutenção das características e da imprescindível qualidade na utilização. Os produtos devem ser 
apresentados de maneira que seja assegurada a garantidas características físico-químicas, controle de prazo de validade e a preservação da qualidade. Além disso, o sistema de distribuição deve assegura qualidade e agilidade na entrega de medicamentos até seu destino final, os padrões pré-estabelecidos devem ser cumpridos.

Os POP's e regulamentos para o armazenamento de medicamentos assessoram no trabalho diário e necessitam ser elaborados com a participação do farmacêutico, e devem ser atualizados sempre que necessário. Devem ser realizados treinamentos periódicos com os funcionários com o objetivo de capacitar e estimular o trabalho possibilitando a integração entre os profissionais, além de criar um ambiente favorável para práticas seguras (ANACLETO et al., 2006).

Em relação à distribuição de medicamentos a maioria dos municípios não possui transporte próprio para execução desta atividade, havendo a necessidade de aguardar a disponibilidade de veículos de outros setores.

A distribuição de medicamentos deve garantir a agilidade na entrega, de maneira rápida e segura, evitando atrasos e desabastecimento ao sistema; assegurar que os produtos cheguem com qualidade ideal e quantidade esperada; garantir que o transporte esteja em condições adequadas de segurança. (MARIN, 2003).

Ao avalia como é realizada a dispensação, pode-se observar que a maioria dos municípios não realiza orientação farmacêutica adequada aos pacientes, além disso, não possui atendimento farmacêutico individualizado.

A dispensação correta garante que o medicamento seja entregue ao paciente, na quantidade necessária, na dose prescrita, e que seja realizada todas as orientações para o uso correto. (ORGANIZACIÓN MUNDIAL DE LA SALUD, 1993). Orientação farmacêutica é realizada pelo farmacêutico e tem por objetivo proporcionar orientação correta para o uso dos medicamentos, identificando o conhecimento já obtido anteriormente pelo paciente sobre sua farmacoterapia promovendo educação nos pontos onde é encontrado algum problema. (CASTRO, 2004; CHEMELLO, 2006)

Como pode ser observado na tabela $3,58,3 \%$ dos municípios tem uma população de 15000 habitantes, nesta mesma proporção se encontra a quantidade de farmacêuticos ligados á Assistência Farmacêutica, tendo em vista que a maioria dos municípios possui apenas 1 farmacêutico ligado a Assistência Farmacêutica. Com isso é possível perceber que não existe farmacêutico responsável por cada posto de distribuição de medicamentos nos municípios entrevistados. 
Veber, et al (2011) encontraram na estruturação dos serviços de assistência farmacêutica debilidade, a pouca atuação de farmacêuticos na dispensação de medicamentos, foram uma delas.

O projeto de Lei PLS no 62/2011, torna obrigatória a presença de farmacêuticos em unidades do Sistema Único de Saúde (SUS), que realizam dispensação de medicamentos.

Tabela 3. Análise do porte dos municípios vinculados a microrregião de saúde de Vitória da Conquista, farmacêuticos ligados a Assistência Farmacêutica e pontos de dispensação de medicamentos.

\begin{tabular}{|c|c|c|}
\hline Variáveis & $\mathbf{N}$ & $\%$ \\
\hline \multicolumn{3}{|l|}{ Porte do Município } \\
\hline Até 15.000 habitantes & 7 & 58,3 \\
\hline De 15.001 a 25.000 habitantes & 1 & 8,3 \\
\hline De 25.001 a 50.000 habitantes & 3 & 25,0 \\
\hline Maior que 100.000 habitantes & 1 & 8,3 \\
\hline \multicolumn{3}{|l|}{ Farmacêuticos ligados a A.F } \\
\hline 1 & 7 & 58,3 \\
\hline 2 & 2 & 16,7 \\
\hline 3 & 2 & 16,7 \\
\hline 13 & 1 & 8,3 \\
\hline \multicolumn{3}{|c|}{ Farmácias/Pontos de Dispensação de Medicamentos } \\
\hline 3 & 1 & 8,3 \\
\hline 4 & 2 & 16,7 \\
\hline 5 & 3 & 25,0 \\
\hline 7 & 1 & 8,3 \\
\hline 8 & 1 & 8,3 \\
\hline 11 & 1 & 8,3 \\
\hline 14 & 1 & 8,3 \\
\hline 17 & 1 & 8,3 \\
\hline 42 & 1 & 8,3 \\
\hline Total & 12 & 100,0 \\
\hline
\end{tabular}

Fonte: Pesquisa própria

Neste contexto a pesquisa também aponta para uma sobrecarga no trabalho do farmacêutico atuante na Assistência Farmacêutica na maioria dos municípios, tendo em vista que existe apenas um farmacêutico responsável por todas as ações e serviços da Assistência Farmacêutica. Em paralelo a isso a maioria dos municípios só dispõe de auxiliar de farmácia na 
farmácia central ou na $\mathrm{CAF}$, sendo que a maioria destes não possui o curso de atendente de farmácia nem capacitação interna.

Com as mudanças na prática farmacêutica à necessidade de formar equipes de auxiliares de farmácia bem capacitados no apoio aos farmacêuticos na dispensação de medicamentos tem crescido, tendo em vista que a falta de tempo do profissional farmacêutico é apontada constantemente como um dos principais impasses para o uso racional de medicamentos. (FARINA, 2009)

Em relação à capacidade para implementar ações de farmacovigilância no município a grande maioria não existe profissionais capacitados para implantar a notificação espontânea de eventos adversos a medicamentos.

Ivama e Souza (2005) (conferem que a farmacovigilância é um campo de interação de grande importância na regulação de medicamentos, contribuindo para o uso racional de medicamentos, tendo em vista que proporciona a identificação precoce de problemas de segurança desconhecidos, apontando e quantificando fatores de riscos promovendo o uso seguro de medicamentos.

\section{Considerações Finais}

No estudo foram evidenciados importantes problemas na gestão da Assistência Farmacêutica. O conjunto que envolve a seleção, programação, aquisição, distribuição e dispensação de medicamentos, necessita subsidiar grandes melhorias para que a assistência farmacêutica nos municípios seja consolidada.

Com está analise é possível notar que apesar dos grandes avanços ocorridos ao decorrer dos anos com a consolidação do SUS, particularmente na Assistência Farmacêutica, o armazenamento de medicamentos ainda ocorre de maneira que não dispõe de requisitos essenciais para manter a qualidade do medicamento.

Os achados evidenciam que existem inviabilizações na dispensação e na atenção farmacêutica, uma vez que os municípios encontram grandes dificuldades no exercício de atividades voltadas para a garantia do uso racional de medicamentos. A Assistência Farmacêutica ainda é vista como sendo apenas um setor responsável pela entrega de medicamentos, onde o enfoque maior é a aquisição e distribuição de medicamentos 
A pesquisa deixa exposta as deficiências no cumprimento das ações relacionadas à Assistência Farmacêutica por parte dos municípios. E indispensável à conscientização dos gestores de todas as esferas, a respeito da importância da organização e estruturação da Assistência Farmacêutica. Investimentos necessitam ser conduzido para melhorias na estrutura física das unidades de dispensação de medicamentos e na capacitação dos funcionários, promovendo acessibilidade ao medicamento e o seu uso racional, levando a uma resolubilidade do sistema de saúde.

\section{Referências}

ANACleto, T. A.; PERINE, E.; ROSA, M. B. Prevenindo erros de dispensação em farmácias hospitalares. Infarma, v.8, $\mathrm{n}^{\circ} 7 / 8$, 2006. Disponível em: <http://www.cff.org.br/sistemas/geral/revista/pdf/13/inf32a36.pdf>.Acessado em: 20 de outubro de 2017.

BARRETO, J. L.; GUIMARÃES, M. C. L. Avaliação da gestão da Assistência Farmacêutica básica em municípios baianos, Brasil. Cad. Saúde Pública, v. 26, n. 6, p. 1207-1220, 2010.

BRASIL. Decreto $n^{\circ}$ 68.806, de 25 de junho de 1971. Institui a Central de Medicamentos (CEME). Diário Oficial [da] República Federativa do Brasil, Brasília. 26 jun. 1971

BRASIL. Presidência da Republica. Casa Civil. Bubchefia para Assuntos Jurídicos. Aprova Consolidação das Leis de Trabalho. 1 de Maio de 1943.

BRASIL. Casa Civil. Subchefia para Assuntos Jurídicos. Lei n 8.080, de 19 de setembro de 1990. Dispõe sobre as condições para a promoção, proteção e recuperação da saúde, a organização e o funcionamento dos serviços correspondentes e dá outras providências. Diário Oficial da União, 17 de setembro de 1990.

BRASIL. Ministério da Saúde. Portaria GM N 3.916, de 30 de outubro de 1998. Portaria de aprovação da Política Nacional de Medicamentos. Brasília: Diário Oficial da União; 1998.

BRASIL. Portaria GM No. 176, de 8 de março de 1999. Estabelece critérios e requisitos para a qualificação dos municípios e estados ao incentivo à Assistência Farmacêutica Básica e define valores a serem transferidos. Diário Oficial [da União], Brasília; 8 mar. 1999.

BRASIL. Ministério da Saúde. Assistência Farmacêutica na Atenção Básica: instruções técnicas para sua organização. Brasília/DF, 2002)

BRASIL. Ministério da Saúde. Resolução n ${ }^{\circ}$ 338, de 06 de maio de 2004. Política Nacional da Assistência Farmacêutica. Diário Ofícial da União, 06 de maio de 2004. BASILE, R. P. A importância da assistência farmacêutica, Portal Educação, 2008. Disponível em: 
<http://www.portaleducacao.com.br/farmacia/artigos/119/a-importancia-da-assistenciafarmaceutica.> Acessado em 22 de outubro de 2017.

BRUS, S.F et al. Gestão da assistência farmacêutica em municípios do estado da Paraíba (PB): olhando a aplicação de recursos públicos. Rev. Adm. Pública - Rio de Janeiro. 48(3):745-765, maio/jun.2014.

CAMPENSE, M. Proposta para ensino da Atenção Básica na Farmácia. 2006. 126f. Monografia (Residência Multiprofissional em Saúde da Família) Universidade Federal do Paraná. Curitiba.

CASTRO, M.S. Atenção farmacêutica: efetividade do seguimento farmacoterapêutico de pacientes hipertensos não-controlados. Porto Alegre: UFRGS, 2004. 183 p.

CHEMELlO, C.; CASTRO, M. S. Adaptação de método de orientação de pacientes sobre medicamentos por uma análise de compreensão. Acta Farmacêutica Bonaerense, Buenos Aires, v. 25, p. 613-618, 2006

FARINA, S.S., ROMANO-LIBER, N.S. Atenção farmacêutica em farmácias e drogarias: existe um processo de mudança? Rev. Saúde e Sociedade. 2009; 18(1):7-18.

FRAGA, F.N.R. A utilização de um modelo lógico para a reorientação dos serviços farmacêuticos no âmbito municipal [Dissertação de Mestrado]. Porto Alegre: Faculdade de Farmácia, Universidade Federal do Rio Grande do Sul; 2005.

IVAMA, A.M., SOUZA, N.R. A Importância da farmacovigilância: Monitorização da Segurança dos Medicamentos. Revista Revista Fármacos e Medicamentos Instituto Racine, 2005, 34(6):82-88

MAYORGA, P.et al. Assistência farmacêutica no SUS: quando se efetivará? In: Misoczky MC, Bordin R, organizadores. Gestão local em saúde: práticas e reflexões. Porto Alegre: Dacasa Editora; 2004. p. 197-215

MARIN, N., LUZIA, V.L., OSÒRIO, C.G.S., MACHADO, S., organizadores. Assistência farmacêutica para gerentes municipais. Rio de Janeiro: Organização Pan-Americana da Saúde/Organização Mundial da Saúde; 2003.

NASCIMENTO Jr, J.M. Avaliação da assistência farmacêutica na rede publica municipal de Florianópolis/SC [Dissertação de Mestrado]. Florianópolis: Departamento de PósGraduação em Saúde Pública, Universidade Federal de Santa Catarina; 2000.

ORGANIZACIÓN MUNDIAL DE LA SALUD. El papel del farmacéutico em el Sistema de Atención de Salud. Tokio: OPS/HSS/HSE/95.1, 1993.

OPAS. Organização Pan-Americana de Salud. El papel del farmacéutico en el sistema de atenciónde salud. Informe de La Reunión de la OMS Tokio, Japón, 31 de agosto al 3 de septiembre de 1993.Tokio: Organização Pan-Americana de Salud, 1993. 
OPAS. Organização Pan-Americana da Saúde/Organização Mundial da Saúde. Avaliação da assistência farmacêutica no Brasil. Brasília: Organização Pan-Americana da Saúde, 2005.

VIEIRA, F.S., ZUCHI, P. Distorções causadas pelas ações judiciais à política de medicamentos no Brasil. Rev Saúde Públ. 2007;41(2):214-22.

World Health Organization (WHO). How to investigate drug use in Health Facilities. Geneva; 1993.

Como citar este artigo (Formato ABNT):

TEIXEIRA, Silvana R.; TELES, Maauro F.; MOREIRA, Pablo M.B. Avaliação do Perfil da Assistência Farmáceutica dos Munícipios do Sudoeste da Bahia. Id on Line Revista Multidisciplinar e de Psicologia, 2017, vol.11, n.38, p. 454-467. ISSN: 1981-1179.

Recebido: 01.10.2017

Aceito: 03.11.2017 